\title{
Computer-Aided Rehabilitation for the Carpal Tunnel Syndrome using Exergames
}

\author{
${ }^{1}$ Ioannis Pachoulakis, ${ }^{2}$ Diana Tsilidi, ${ }^{3}$ Anastasia Analyti \\ ${ }^{1,2}$ Department of Informatics Engineering, Technological Educational Institute of Crete \\ Heraklion, Crete, Greece; \\ ${ }^{3}$ Institute of Computer Science, Foundation for Research and Technology - Hellas (FORTH) \\ Vassilika Vouton, Heraklion, Crete, Greece; \\ ip@ie.teicrete.gr; dtsilidi@gmail.com; analyti@ics.forth.gr
}

\begin{abstract}
Carpal tunnel syndrome (CTS) has reached epidemic proportions as the surgical release of the transverse carpal ligament is included in the top ten most common operations, which significantly affects health care costs. Additional costs to consider include time off work, lost wages, and diminished workplace productivity. Non-surgical management of CTS such as splinting, non-steroidal antiinflammatory medication, steroid injections and ergonomic modification of the work habits can help in early cases, but can be ineffective in more advanced cases, often leading to recommendation for surgical treatment. It is possible to attain meaningful physiotherapy results through interesting and engaging computer-based games which provide the motivation to continue therapy at home, even away from direct therapist supervision. Motivation and immersion in the game scenario also helps patients forget that they are performing exercises as a part of their therapy. Accordingly, we present a Unity3D Fly-A-Plane game, the scenario of which combines CTS-specific physiotherapy exercises in a natural game scenario to fly an airplane through a sequence of hoops in the sky. The game employs the Leap Motion sensor, whose detailed wrist and hand (including fingers) tracking abilities make it an excellent hardware platform for rehabilitation oriented exercises intended for patients suffering from CTS.
\end{abstract}

Keywords: Carpal tunnel syndrome; Traditional Physiotherapy; Computer-aided rehabilitation; Gamebased Rehabilitation.

\section{Introduction}

Carpal tunnel syndrome (CTS) is a very common and frequent focal peripheral neuropathy in which the median nerve is compressed where it passes through the wrist. CTS commonly causes swelling, pain, tingling and loss of strength in the wrist and hand. Usually the tendons of the wrist become swollen and compress the nerve. The functionality of the median nerve is to control some of the muscles that make the thumb move and transfer sensory information from the thumb and fingers back to the brain [1]. Compressing the median nerve may feel painful, aching, tingling or it may cause numbness in the affected hand. During the night hours the symptoms may deteriorate the quality of the sleep and can become more obtrusive in the morning hours, after waking up. Aching and tingling can be temporarily relieved by hanging the hand out of bed or shaking it around.

Activities like typing on the computer, handwriting or some types of housework may intensify the symptoms, even if the problem is not so perceptible during the day. In some cases CTS symptoms may 
resemble those for other conditions, such as arthritis or disc problems [2], a confusion that can be alleviated with the help of a nerve conduction tests. The syndrome is three times more common in women compared to men, while it affects almost 3\% of the adult population. Persons who tend to perform repetitive wrist movements (especially true in the developed countries) are more prone to develop CTS. For example, CTS-related costs in the U.S. mount to over 2 billion annually, partially due to the high cost of the medical treatment for roughly $3.7 \%$ of the general public who suffer from it but also due to the missed work days. By comparison, in France, 127,269 patients over 20 years were operated to treat CTS in 2008. The syndrome is reported to affect more frequently mostly females between 45 and 59 years old, and also mostly females between 75 and 84 years old [1]. Many people, who use a computer daily (for work, education or entertainment), complain about hand paresthesia, a superficial sensation of tingling, burning or numbness of the hand [3]. However, a small percent of these computer users actually meet the clinical criteria for CTS.

Many CTS patients opt for physiotherapy, which involves tendon gliding of the finger flexor tendons and nerve gliding of the median nerve, thereby helping to reduce pain and increase mobility and strength. Additional exercises may be included, depending on the individual case, to increase muscle strength in the hand, fingers and forearm as well as in the trunk and postural back muscles, combined with stretching exercises to improve flexibility in the wrist, hand and fingers. Physiotherapy mainly aims at moderating the CTS-related symptoms in order to allow the patient to maintain the functionality of his hands in everyday activities and obviate the need for surgery. Even if surgery cannot be prevented, post-surgery physical therapy helps to restore strength and full functionality to the hand.

However, the benefits of physical therapy are not instant, but are rather felt in the long-term (many weeks or months). Daily repetition of the prescribed exercises is required to have partial recovery of impaired movement. These sessions tend to be long, tiresome and tedious and may be difficult to schedule in frequent sessions because of healthcare costs [4]. Outpatient therapy gives a solution to this problem, as the patients may perform exercises at home on a regular basis, while communicating remotely with the therapist, so that a physical therapist may not be required to be present after the first few sessions.

Even at home, treatment requires time-consuming and repetitive exercises that are not very pleasant for the patients and end up monotonous and boring, resulting in only $31 \%$ of people performing them as recommended [5]. In addition, since the presence of the physical therapist is not necessary for the performance of the exercises, it is natural for the patients to lose their motivation and confidence, while doing them alone at home, especially without immediate significant improvement to be expected. These factors allow many patients to neglect exercising on a regular basis and abandon the treatment before it is completed. Therefore, it is important to provide patients who suffer from motor disabilities rooted in neurological and musculoskeletal conditions appropriate and engaging rehabilitation programs designed to improve their quality of life. A number of studies [5], [6], [7], [8], conclude that interactive games have the potential, through several of their elements, to enhance the results of rehabilitation and therapy.

The idea of attaining meaningful physiotherapy through interesting and engaging games is promising, since it can provide the motivation to continue therapy at home, even when they are not under direct therapist supervision. Accordingly, serious games have started to be used in physiotherapy and rehabilitation, as they can promote healthy habits and exercise, while entertaining and rewarding the players [9]. Motivation and immersion in the game scenario also helps patients forget that they are performing exercises as a part of their therapy. The key factor is that exergames can employ immersive game scenarios to promote repetitive actions. In addition, goal-oriented and highly interactive games, 
I oannis Pachoulakis, Diana Tsilidi, Anastasia Analyti; Computer-Aided Rehabilitation for the Carpal Tunnel Syndrome using Exergames, Advances in Image and Video Processing, Volume 6 No 2, April (2018); pp: 44-56

can provide sufficient motivation to perform and complete their exercises at home. Typical game features like controlling 3D worlds and elements like high scores, achievements, rewards and positive feedback can make therapy fun and addictive and help maintain interest and, as a result, play more and help to speed up recovery. In doing that, exergames create an environment where repetition is an advantage and something to be enjoyed rather than dreaded [10].

The present work discusses the key advantages of the Leap Motion sensor that make it a promising tool on which to base the creation of CTS-oriented serious games. These advantages have been explored through custom CTS-oriented interactive serious games which require users to perform CTS-specific exercises (extension/flexion and radial/ulnar deviation) to successfully complete the game objectives.

\section{Traditional CTS Physiotherapy}

When the wrist and the fingers are extended the median nerve is displaced farthest under the transverse carpal ligament into the hand. The study of McLellan and Swash [11] described how the median nerve slides distally by $10-15 \mathrm{~mm}$ relatively to a fixed bony landmark in the carpal tunnel, when the wrist is hyper-extended, while the displacement mentioned above, which occurs during the flexion of the wrist and the fingers, is often four times greater at the wrist that in the arm. Additionally, the nerve is getting displaced $11 \mathrm{~mm}$ distally during the active and passive extension of the wrist and the fingers and $4 \mathrm{~mm}$ proximally during the flexion. Local stretching of the median nerve, that would normally occur, is prevented by the longitudinal sliding. Szabo et al. [12] have also shown the differences between median nerve and digital flexor tendon excursion in the carpal tunnel and the linear relationship that exists between median nerve movement and that of the flexor tendons. Recurrent active wrist and digital flexion and extension exercises reduce the pressure within the carpal tunnel. Thus, it is possible to affect the clinical course of CTS of some patients by using a specific series of exercises. A systematic procedure, which actively forces the median nerve and the flexor tendons to their maximal excursion through the carpal tunnel, is proposed in case that the attachment to the median nerve exists preoperatively. Certain studies have also proposed a regimen suitable for postoperative nerve gliding that is used to minimize scar adhesions and maximize nerve excursion through the carpal canal, which is reported to be effective in the management of postoperative CTS, with relief of pain and low recurrence rates. The performance of these nerve and tendon gliding exercises significantly affects symptom resolution by stretching the adhesions in the carpal canal, broadening the longitudinal area of contact between the median nerve and the transverse carpal ligament, also by reducing tenosynovial edema by a "milking action", thus improving venous return from the nerve bundles and finally reducing pressure inside the carpal tunnel. This alternative conservative method of CTS management reduces the need for a surgical intervention.

These neuromobilization techniques may be applied when there is certain evidence of an entrapment of the median nerve at the wrist and the neurodynamic test is positive. These techniques combine repetitive movements of the hand that provoke the symptoms and movements in the more distal and proximal segments. The position and the movements necessary for the neurodynamic test are similar to those needed for the treatment exercises, which usually include movements performed in a moderate pace divided in 3 sets of 10 repetitions in each set and a 3 second hold at the final stretched position. The therapist can decide to focus on a specific part of the nerve, if the test shows that this part is responsible for the symptoms, but without abandoning the exercises for the other parts of the nerve. Especially in the first few sessions the patients may experience a feeling of "stretching", tissue tension, light numbness, even a slight increase of pain symptoms during the technique; however the symptoms decrease or completely stop immediately following the procedure. The patient has to 
perform daily home exercise program including self-mobilization techniques for the median nerve, which can be performed in standing or sitting position.

Determining whether the condition of CTS is mild, moderate, or severe can clarify and restrict intervention choices. Before applying the specific therapy it is important to assess the severity of the symptoms, as a patient with a mild CTS condition may have a successful symptom reduction by only using splints at night and by altering the activities during the day, while a patient with moderate or severe CTS will not usually benefit so much from splint usage. Recent practice links the individual differences in CTS symptoms or the results of therapy to a genetic predisposition for developing the syndrome. As a result, people with the same activity or the same treatment might have different symptoms and treatment results.

The most common exercises recommended for the conservative management of the symptoms of CTS are tendon gliding of the finger flexor tendons and nerve gliding of the median nerve [13]. Studies that included measurements of the carpal tunnel pressure have shown that occasional exercise of active wrist and finger motion lasting about one minute lowers the pressure within the carpal tunnel [14]. The gliding exercises include moving the fingers in a specified pattern of exercises and help the tendons and nerves glide more smoothly through the carpal tunnel. While there's some evidence that gliding exercises can help relieve symptoms when used alone, these exercises appear to work better in combination with other treatments, such as splinting. Also manual therapy integrates a patented form of instrument-assisted soft tissue mobilization that gives the opportunity to the therapists to accurately detect and treat scar tissue and restrictions that effect normal function.

\section{Computer-aided, CTS-oriented exergames}

Serious games, a special category of games, are commonly used in training in the military, health care or engineering, but can be also a very useful tool for education. Since the games usually involve challenges and rewarding systems, they can motivate users to perform a number of tasks for the purpose of the game they would not normally undertake on their own. Accordingly, serious games find applications in health care and help mitigate severe mobility impairments due to neurological and musculoskeletal problems in multiple sclerosis and stroke sufferers. In addition, game-based physiotherapy affords patients that require regular physical therapy sessions to regularly practice at home and keep their interest, especially when results are not prompt. Accordingly, it is important to build exergames that both motivate patients [15] and facilitate the completion of repetitive, tedious tasks efficiently, so as to remain dedicated to the program and enjoy better recovery prospects [16]. In the case of CTS, the commonly prescribed gliding exercises, which are repetitive movements of the wrist and fingers can be successfully implemented in an engaging game environment with many interesting features keeping the patient amused and motivated to attain higher levels of performance.

The last decade has seen a number of attempts to take advantage of the emerging motion sensor technology in the fields of physiotherapy and rehabilitation [17], [18], and [19]. Although approaches based on academic research [20], [21] present game prototypes using sensors for physiotherapy treatment, there are also commercially available products. VirtualRehab-Hands [22] is a mini-game platform developed by Virtualware that uses the Leap Motion controller to offer physiotherapy for conditions such as Parkinson's disease and stroke. Ten Ton Raygun's Visual Touch Therapy software [9] also employs the Leap Motion controller to make therapy fun and engaging, while allowing the monitoring and assessment by therapists. The target group of these therapeutic games is mostly poststroke patients, but also individuals with spinal cord injuries, head injuries and nerve damage. Exactly because of its accuracy and portability, the Leap Motion sensor is very promising for CTS rehabilitation. 


\subsection{The Leap Motion Sensor}

The Leap Motion controller presents a new gesture and position tracking system with accuracy that reaches a sub-millimeter. This sensor is a small USB peripheral device which is designed to be placed on a table, facing upward. The device observes an approximately hemispherical volume to a distance of about 1 meter from it using two monochromatic IR cameras and three infrared LEDs. The LEDs generate IR light and the cameras generate almost 300 frames per second of reflected data, which is streamed through a USB cable to the host computer to be analyzed by the Leap Motion controller software to generate 3D position data for the arm, hand and fingers.

The assessment of optical 3D sensors usually depends on their stereo vision cameras, the structured light sensors and the time of flight cameras. The stereo vision cameras that are formed by two 2D optical cameras determine the depth of the environment by comparing and combining the points extracted by the two cameras. Structured light sensors inspect the distortion of a noted design to an unfamiliar area to ascertain the shape of the objects in the three dimensional space, while the so called time of flight (TOF) 3D cameras resolve the distance of the target object by measuring the time of flight of a light signal emitted by the device. Also, the devices can be divided into photonic mixer devices, which measure the phase shift between the sent infrared light beam and the reflected returned light, and laser sensors, which measure the distance to an object according to the time the reflected pulsed laser beams need to return to the sensor.

The Leap Motion controller can be classified as an optical tracking system based on stereo vision, as it emits infrared light and has two infrared cameras that calculate the position points of the target objects, such as hands, fingers or tools in the Cartesian space. The calculated position is relative to the Leap Motion controller's center point that is located at the position of the second, centered infrared emitter, as can be seen in Error! Reference source not found..
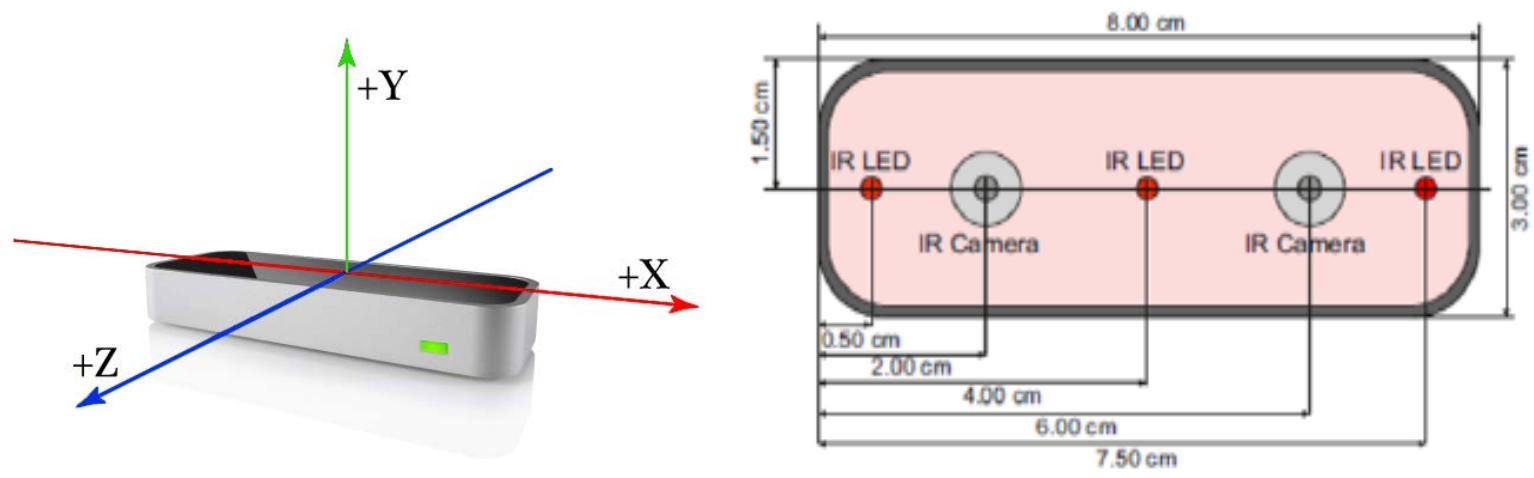

Figure 1: The Leap Motion Sensor on the left and schematic view of its internals on the right

Weichert, Bachmann, Rudak and Fisseler [23] evaluated the accuracy and repeatability of the Leap Motion controller with the use of industrial robots. The conclude that the deviation between the certain object position in the three dimensional space and that calculated by the Leap Motion sensor is less than $0.2 \mathrm{~mm}$ with repeatability at the level of less than $0.17 \mathrm{~mm}$. Other popular sensors, like Microsoft Kinect, tested in the experiment could not achieve such accuracy results. While it is not possible to achieve the theoretical accuracy of $0.01 \mathrm{~mm}$ in real conditions, still the sensor offers an overall accuracy of about $0.7 \mathrm{~mm}$, which is considered high precision for any hand gesture recognition user interfaces and thus makes the Leap Motion controller appropriate for physiotherapy applications. 
Tracking data transmitted from the sensor to the application is in the form of snapshots called frames, which contain calculated position and rotation points and other information regarding the entities that were detected by the sensor at a certain moment of the snapshot. The physical entities that can be detected by the sensor are the hands and the fingers of the user or in some cases tools like pens or pencils.

The frame() function of the Controller class can be used to retrieve a frame object containing an entity's tracking data. This function utilizes a history parameter that specifies the number of previous frames to obtain, while the history buffer can fit the last 60 frames. Further data of the frame class can be accessed through several class functions.

The data of a detected hand of a user can include information about the position, the rotation, the direction and the motion of the hand:

- isRight, isLeft - identifies the detected hand (right or left).

- Palm Position - calculates the position of the center of the palm in millimeters relatively to the sensor's origin.

- Palm Velocity - the speed and direction of the palm in millimeters per second.

- Palm Normal - represents a vector perpendicular to the plane of the palm of the hand which points out of the palm.

- Direction - a vector pointing from the center of the palm toward the fingers.

- grabStrength, pinchStrength - variables that describe the posture of the hand.

The motion factors provide relative translation, rotation and scale differentiations in movement between different frames. In addition, the vector class includes methods that retrieve the pitch (rotation around the $x$-axis), yaw (rotation around the $y$-axis), and roll (rotation around the z-axis) rotations of the detected entity [24].

\subsection{The Unity3D Game Engine}

Unity3D is a popular cross-platform game development tool commonly used for the development of PC, consoles, mobile devices video games and applications. This game engine focuses on portability, targets a number of APIs and allows publishing the resultant applications in Android, Apple TV, BlackBerry 10, iOS, Linux, Nintendo 3DS, OS X, PlayStation 4, PlayStation Vita, Unity Web Player, Wii, Wii U, Windows Phone 8, Windows, Xbox 360 and Xbox One [25]. Unity's versatility includes the ability to determine the appropriate texture compression and resolution settings for each platform that it supports. In addition, the game engine provides reflection, bump, and parallax mappings, screen space ambient occlusion (SSAO), dynamic shadows using shadow maps, render-to-texture and full-screen post-processing effects. The graphics engine recognizes the most compatible modification of the shader regarding the video hardware of the system, allows alternative specifications for the shader [26] and includes an asset server and Nvidia's PhysX physics engine.

Within a simple user interface Unity3D's integrated editor shown in Figure 2 provides external editors for scripting, texturing and model editing, as well as a convenient asset and file organization system. The game engine is also highly optimized so it can support high resolution meshes without a significant frame rate decrease. In addition, assets can be imported and for textures, models and scripts all common file formats are supported. 


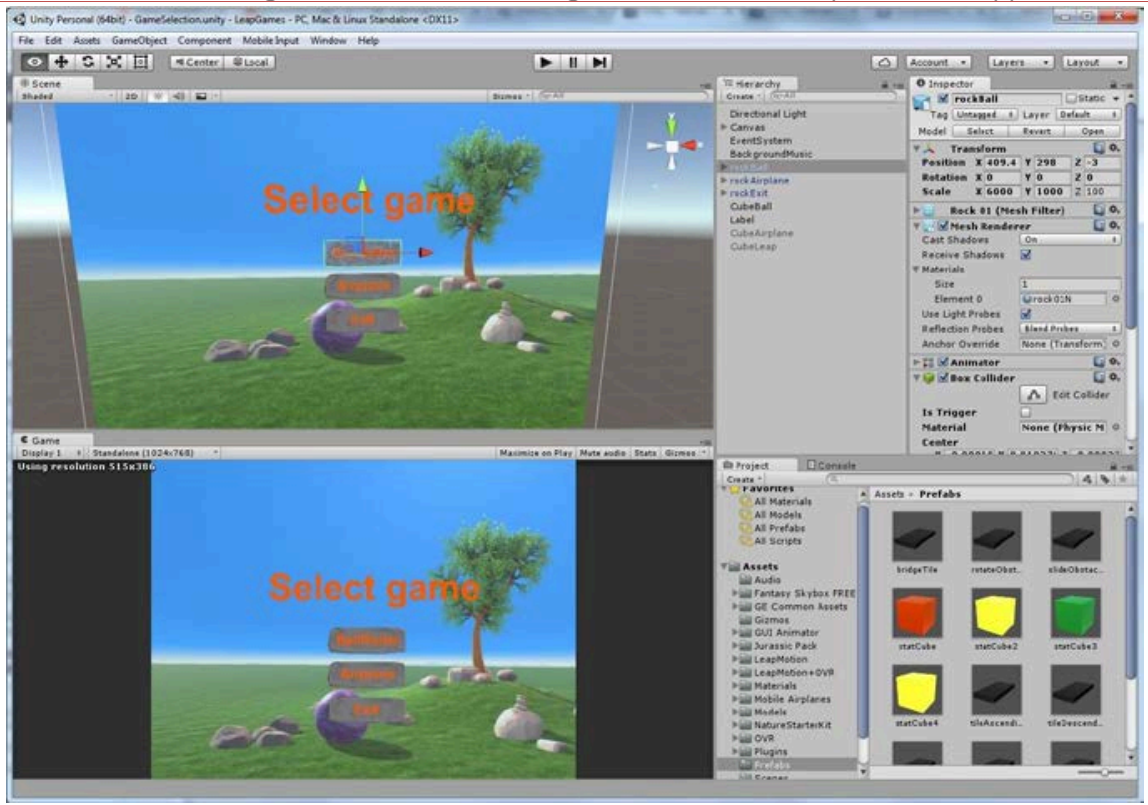

Figure 2: Developing an exergame in Unity3D for computer-aided rehabilitation of the Carpal Tunnel Syndrome.

Unity's shader system is usable and flexible; it includes shaders ranging from very basic like Diffuse or Glossy to advanced, like self-illuminated bumped specular etc. The built-in shaders can be successfully combined with any type of light. On the other hand it is possible to create custom shaders by using ShaderLab language with Cg and GLSL. The terrains of Unity are not particularly demanding in terms of hardware and perform satisfactorily on lower-end hardware, as long as the models contain a reasonable amount of polygons. The lights and the shadows available in Unity3D include real-time softshadows, baked light maps, halos and lens flares.

Unity3D also contains a network system that allows the development of full featured real-time multiplayer games, since it is able to access HTTP servers and establish connection with PHP websites. Regarding physics, the game engine includes a built-in physics engine named Ageia Phys $\mathrm{X}^{\mathrm{TM}}$, which supports the rigid body paradigm which reacts to the forces being applied as well as collisions and does not require scripting to function at its basic level. The game engine also provides rich libraries and thorough documentation regarding the .NET-based C\# and JavaScript scripting. In addition to the native Unity editor, users may utilize the Visual Studio editor to compile scenes.

\subsection{Customized Exergames for CTS}

The present work augments our existing Leap Motion sensor -based CTS rehabilitation system [27] intended to offer physical therapy opportunities at home combined with the Fly-A-Plane exergame. The process benefited from a number of meetings with a physiotherapist in order to (a) glean details regarding CTS and its treatment and (b) select a number of physiotherapy exercises to be performed by the player (patient) to control game progress. That initial exercise set Error! Reference source not found.Error! Reference source not found.contains ten distinct movements of the wrist and the fingers, including different rotations and postures of the wrist and finger joints. Out of that initial set, the four exercises that were selected to implement to control the Fly-A-Plane game appear in Figure 3. The criterion for the given selection stems from the need that, since the movements of the exercises must correspond to the movements of the avatars in the game environment, the user should naturally perform these exercises without confusion. 


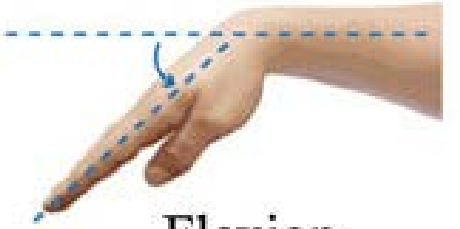

Flexion
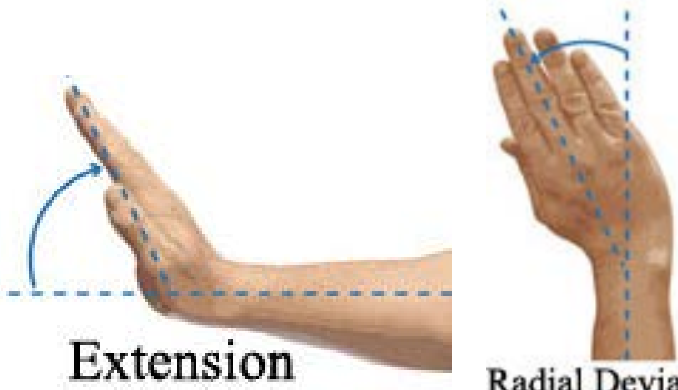

Radial Deviation Ulnar Deviation

Figure 3: - The four exercises selected for implementation in the Fly-A-Plane exergame.

The goal of the Fly-A-Plane exergame is to guide an airplane through a number of hoops in the sky (game scene). If the Leap Motion sensor detects one of the hand movements listed in Figure 3, the airplane is directed to perform a pre-programmed movement corresponding to that particular detection event. Therefore, the physical movement of the player's arm corresponds very naturally to the game avatar (airplane) movement: positive pitch rotation for a wrist extension, negative pitch rotation for wrist flexion, positive yaw rotation for ulnar deviation and negative yaw rotation for radial deviation as shown in Figure 4 and detailed in Table.
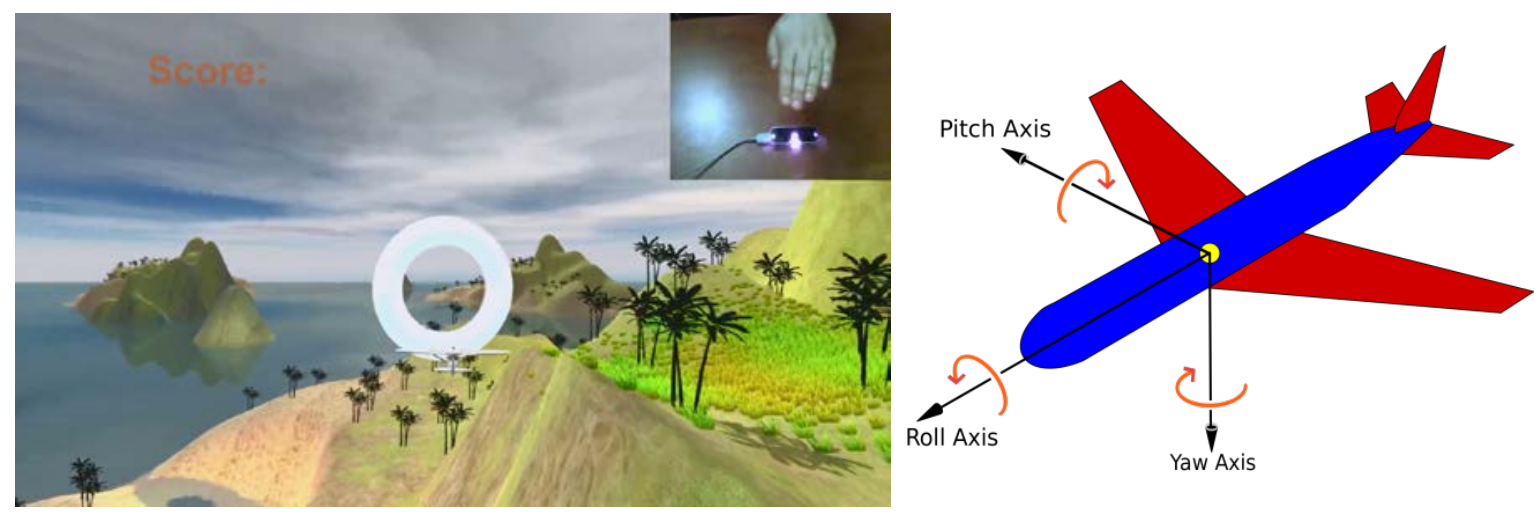

Figure 4: - On the left appears a selected main scene of the Fly-A-Plane exergame. The insert in the top right shows the player's hand above the Leap sensor guiding the plane. The principal axes of rotation of the plane appear in the right figure.

Table 1. Correspondence between physical and game avatar (airplane) movements in the Fly-A-Plane exergame

\begin{tabular}{|c|c|}
\hline Physical therapy exercise movement & Game avatar movement \\
\hline Wrist extension (upward rotation) & Positive pitch angle rotation \\
\hline Wrist flexion (downward rotation) & Negative pitch angle rotation \\
\hline Wrist ulnar deviation (rotation to the right) & Positive yaw angle rotation \\
\hline Wrist radial deviation (rotation to the left) & Negative yaw angle rotation \\
\hline
\end{tabular}

The scene of the Fly-A-Plane game is constantly generated as long as the player guides the airplane in any direction. The scene is created by generating a $3 \times 3$ grid made of the initial terrain, containing exotic islands and sea environment and is repeated in a specific direction from the game starting point, whenever the airplane reaches that area. The need for generating the grid is coded in the Update() method so that it is checked in every frame, and its position is determined in the UpdateTerrainPositionsAndNeighbors() method listed in Figure. 


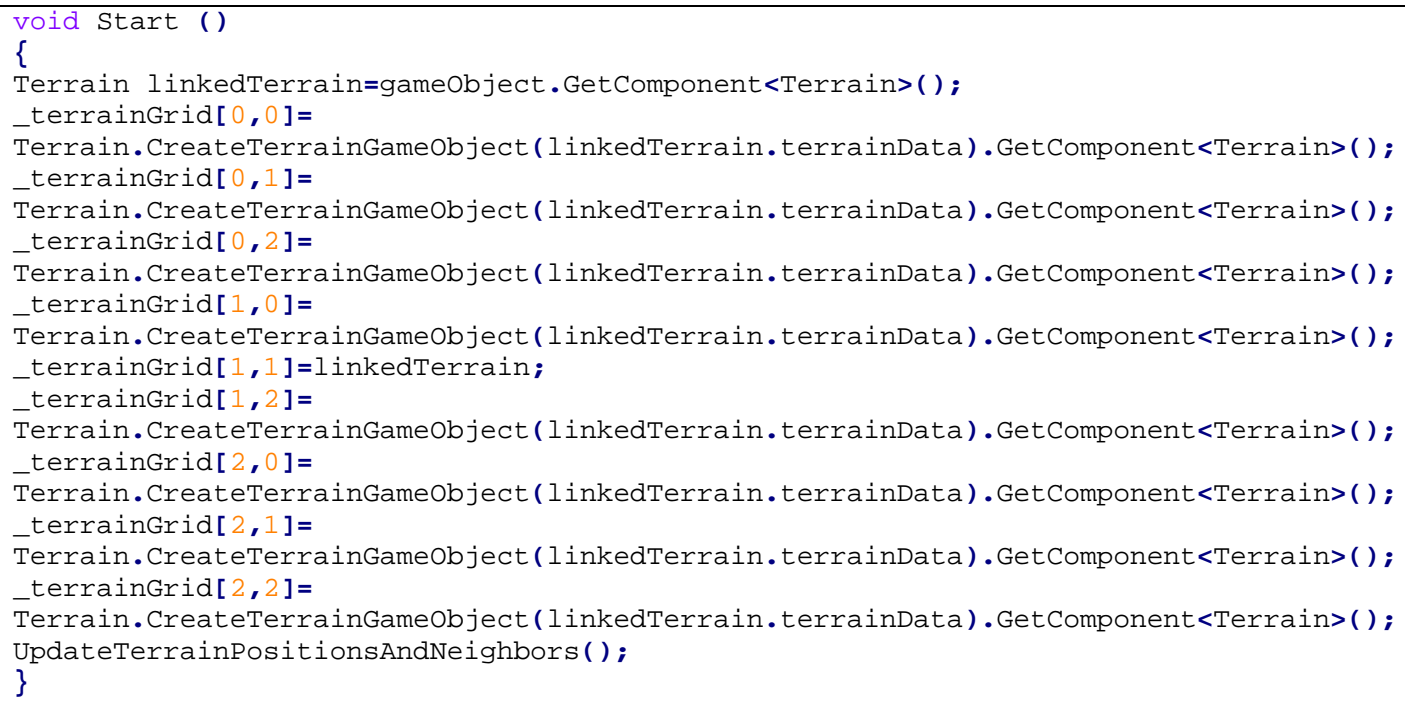

Figure 5: - Sample code responsible for the creation of a 3x3 grid repeating the initial terrain object.

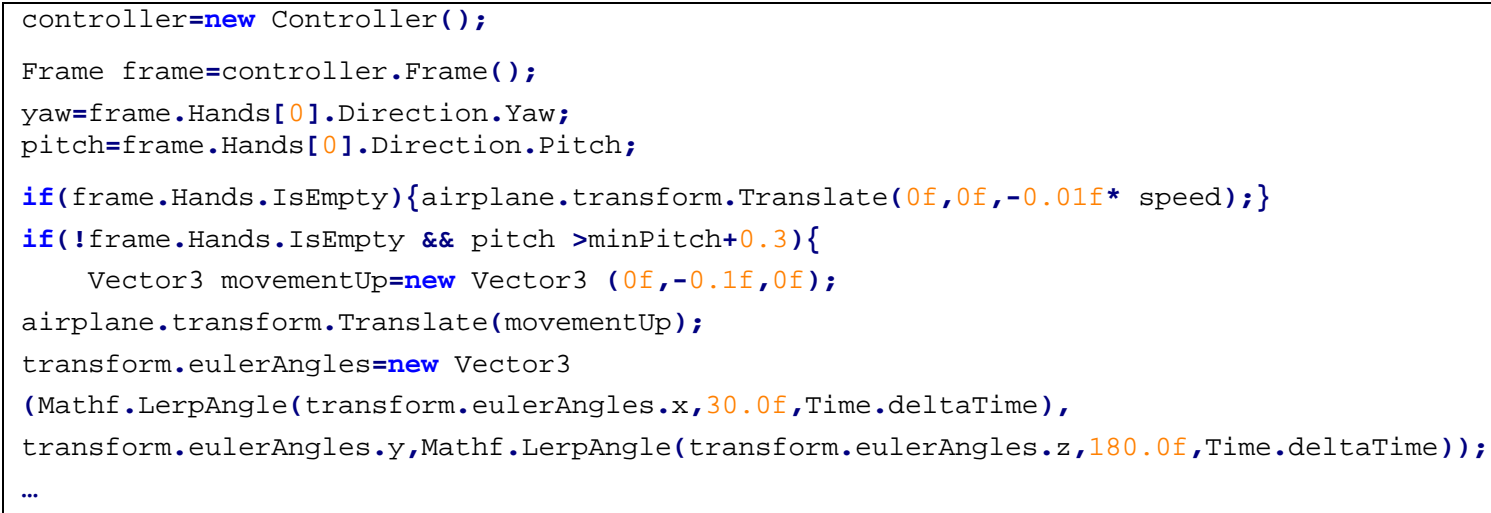

Figure 6: - Sample code implementing the airplane movement controller.

Since the main goal of this game is to fly the airplane through the rings in the game environment, it is exactly the sequence of positions of the rings that is crucial for the correct execution of the physical therapy exercises. For that reason the rings are being instantiated dynamically according to rules implemented according to the directions / requirements stated by the physiotherapist for the particular patient. For example, if the user has just performed an ulnar deviation to turn the airplane to the right to pass through a ring and the script instructs for a radial deviation to be performed next, the new ring must appear to the left of the plane's new direction. Similarly, when the next ring is situated in front of the airplane and at a higher altitude, the user is forced to perform an extension of the wrist to make the airplane to climb to reach that ring, whereas a ring positioned at a lower altitude requires a flexion of the wrist. In this way, the patient naturally executes a prescribed exercise script in a meaningful and engaging game environment. Essentially, this is the job of the airplane movement controller detailed in Figure. 

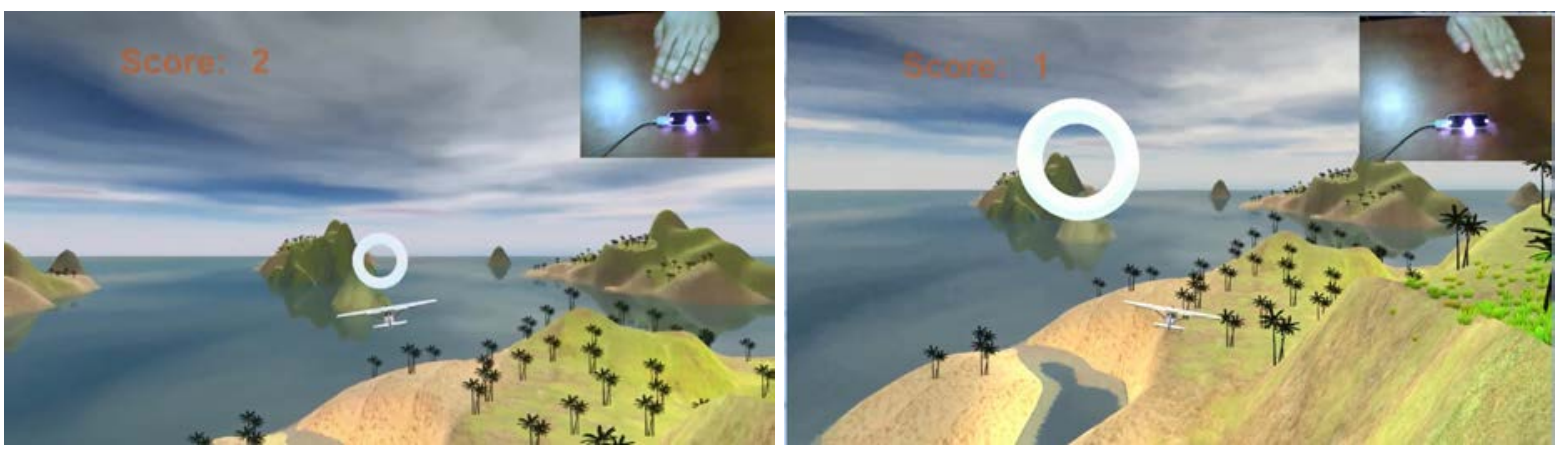

Figure 7: - Left panel: To turn the airplane left requires a radial deviation of the right hand or an ulnar deviation of the left hand. Right panel: To turn the airplane to the right requires an ulnar deviation of the right hand or a radial deviation of the left hand

In the left panel in Figure the next ring to be collected is located in front and to the left of the airplane, forcing the user to rotate the plane to the left. This can be accomplished using a radial deviation of the right hand or an ulnar deviation of the left hand. By comparison, the right rotation of the plane required in the right panel in Figure requires either an ulnar deviation of the right hand or a radial deviation of the left hand. (Each panel shows a mirrored image of the hand in the upper right corner of each panel's game scene shows a mirrored image of the hand above the Leap Motion controller.)

Unlike the first game developed for our CTS-rehabilitation platform (see Roll-A-Ball game in [27]) where wrist flexion and extension were employed as "go" and "stop" actions on the rolling ball, respectively, in the Fly-A-Plane exergame the airplane must be allowed to gain or lose altitude, meaning that exercise patterns detected by the Leap Motion sensor are being mapped onto game actions based on the specific abilities of the controlled game objects. The airplane moves forward constantly, but with a slow speed, so that the game is more challenging, while the user still has enough time to perform the exercise at a normal pace. The insert in the left and right panels in Figure shows the wrist extension / flexion exercise being performed in order to move the airplane up / down, respectively.
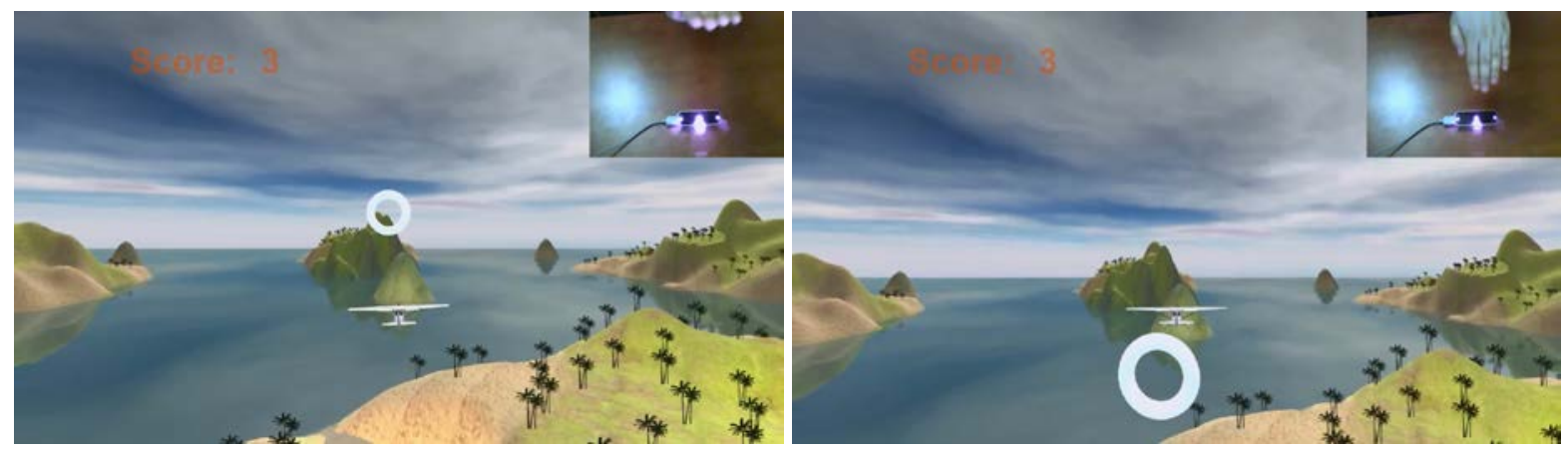

Figure 8: - Left panel: Execution of a wrist extension to cause the airplane to gain altitude; Right panel: Execution of a wrist flexion to cause the airplane to lose altitude..

It is worth noting in Figure 3 that the angular range of normal wrist rotation is not the same in both ulnar and radial deviation in the sense that the range of motion for the ulnar deviation of the right hand or the radial deviation of the left hand is considerably more wide than the possible range of motion for the ulnar deviation of the left hand or the radial deviation of the right hand. Accordingly, the detection limits for the Leap motion sensor were set higher in the former than in the latter case. Recognizing that CTS severity also differs among patients, additional customization is possible and each exercise is parametrized to be adapted to the needs of the individual patient. For demo purposes the system presently allows users to select a predefined difficulty level (easy, medium and hard) for each game they play (shown in the left panel in Figure). A harder difficulty level yields a higher speed of movement 
I oannis Pachoulakis, Diana Tsilidi, Anastasia Analyti; Computer-Aided Rehabilitation for the Carpal Tunnel Syndrome using Exergames, Advances in Image and Video Processing, Volume 6 No 2, April (2018); pp: 44-56

for the avatar and implies more extreme wrist rotation angles. For example, if the user chooses to play in easy mode, the airplane will be moving forward slower and it will require smaller wrist rotations to turn the plane up or down or change its altitude. This can be a level appropriate for a beginner or a more advanced case of CTS.
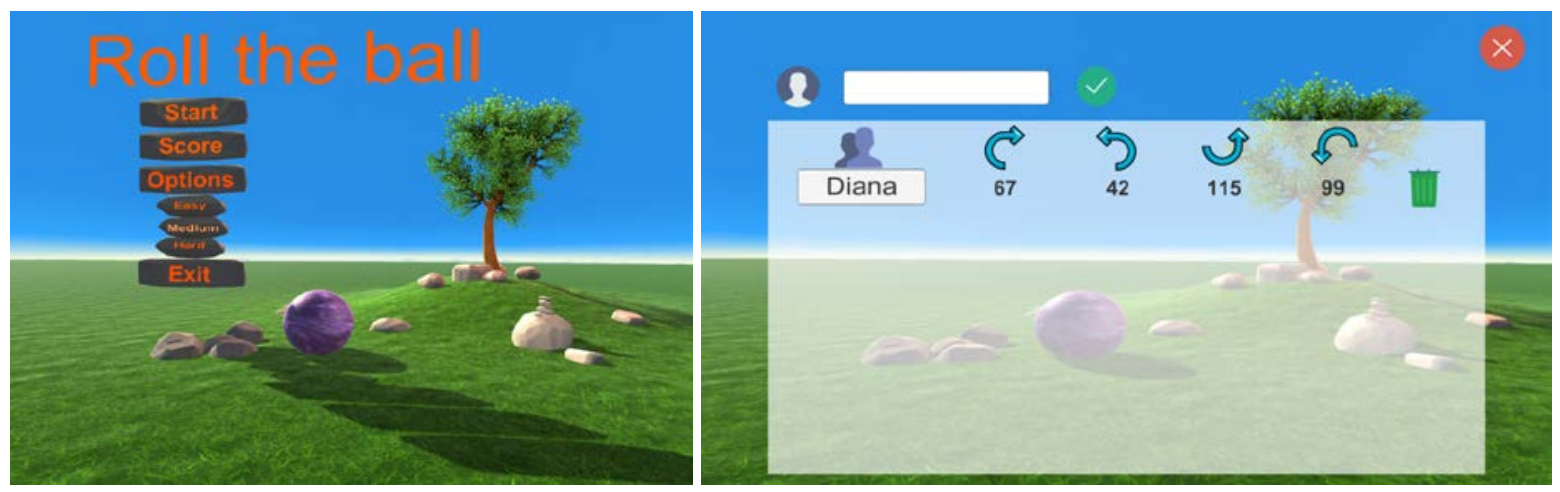

Figure 9: - Left: Top level showing the difficulty levels; Right: Detailed user performance per game cycle..

Finally, the system stores high scores for every hand rotation (right, left, up and down) for every user profile as shown in the right panel in Figure and sends relevant data with ISON (JavaScript Object Notation) to a server, which displays the score graph for every user by the date of the game play.

\section{Discussion and Future Work}

In this work we enrich our Carpal Tunnel Syndrome (CTS) rehabilitation platform [27] with a Fly-A-Plane exergame aimed at alleviating symptoms of the syndrome for suffering patients. The game has been developed Unity3D and capitalizes on the ability of the Leap Motion sensor to accurately track wristhand movement and detect certain pre-programmed rehabilitation movements. At first glance, the use of the Leap Motion sensor may appear limited for rehabilitation, because it tracks only the lower arm, hand and fingers. However this is sufficient for serious games tailored to CTS patients.

One possible direction for future work is to examine whether and to what degree of usefulness the Leap Motion sensor can be paired with Microsoft's Kinect to provide the required precision in the detection of hand gestures like pronation/supination and flexion/extension of metacarpophalangeal and the proximal interphalangeal joints of the hand, while Kinect provides larger scale body tracking.

Finally, technology-enabled rehabilitation can offer measurable results as games can be designed to collect performance data and use these to calculate and store appropriate performance metrics. This possibility should allow therapists to assess long term progress and to mitigate a sense of accomplishment to the patients which can be very beneficial during treatment.

\section{ACKNOWLEDGEMENTS}

The authors thank physiotherapist Mr. George Stratakis for his input in gleaning exercises that are feasible to model using the Leap Motion Sensor.

\section{REFERENCES}

[1] M. Chammas, J. Boretto, L. M. Burmann, R. M. Ramos, F. C. dos Santos Neto, and J. B. Silva, “Carpal tunnel syndrome - Part I (anatomy, physiology, etiology and diagnosis)," Rev. Bras. Ortop. (English Ed., vol. 49, no. 5, pp. 429-436, 2014. 
[2] A. Alexander, "Carpal tunnel syndrome - Information for Physiotherapists," Oxford University Hospitals NHS Trust. 2014

[3] T. Gregory, D. Chamblin, J. Firestone, A. Friadman, H. Chirstopher, P. Douglas, M. Kliot, R. Lawrence, E. Thomas, B. Nicholas, D. Michael, T. Kjerulf, K. O’Bara, S. Carlson, and J. G, “Work-Related Carpal Tunnel Syndrome Diagnosis and Treatment Guideline," Washingtonworkingsolutions. Net. Washington State Department of Labor \& Industries, pp. 1-18, 2017.

[4] M. Abdur Rahman, "Multisensor Serious Game-Based Therapy Environment for Hemiplegic Patients," Int. J. Distrib. Sens. Networks, vol. 11, no. 6, p. 910482, Jun. 2015.

[5] G. Alankus, R. Proffitt, C. Kelleher, and J. Engsberg, "Stroke Therapy through Motion-Based Games," ACM Trans. Access. Comput., vol. 4, no. 1, pp. 1-35, Nov. 2011.

[6] A. Neil, S. Ens, R. Pelletier, T. Jarus, and D. Rand, "Sony PlayStation EyeToy elicits higher levels of movement than the Nintendo Wii: Implications for stroke rehabilitation," Eur. J. Phys. Rehabil. Med., vol. 49, no. 1, pp. 13-21, 2013.

[7] A. M. D. C. Souza, M. a Gadelha, E. a G. Coutinho, and S. R. Santos, "A video-tracking based serious game for motor rehabilitation of post-stroke hand impairment," SBC J. 3D Interact. Syst., vol. 3, no. 2, pp. 3746, 2012.

[8] A. De Graaf, "Gaming Motion tracking technologies for rehabilitation," Study Tour Pixel 2010, pp. 1-6, 2010.

[9] A. Gupta, “AXLR8R Spotlight: Making Physical Therapy Fun with Ten Ton Raygun," 2017. [Online]. Available: http://blog.leapmotion.com/axlr8r-spotlight-making-physical-therapy-fun-with-ten-tonraygun/.

[10] "What if Physical Rehabilitation Were as Easy as Playing a Video Game? - Not Impossible." [Online]. Available: http://www.notimpossiblenow.com/lives/what-if-physical-rehabilitation-were-as-easy-asplaying-a-video-game. [Accessed: 05-Mar-2016].

[11] D. L. McLellan and M. Swash, "Longitudinal sliding of the median nerve during movements of the upper limb," J. Neurol. Neurosurg. Psychiatry, vol. 39, no. 6, pp. 566-570, 1976.

[12] R. M. Szabo, B. K. Bay, N. A. Sharkey, and C. Gaut, "Median nerve displacement through the carpal canal," J. Hand Surg. Am., vol. 19, no. 6, pp. 901-906, Nov. 1994.

[13] S. L. Michlovitz, "Conservative Interventions for Carpal Tunnel Syndrome," J. Orthop. Sport. Phys. Ther., vol. 34, no. 10, pp. 589-600, Oct. 2004.

[14] L. M. Rozmaryn, S. Dovelle, E. R. Rothman, K. Gorman, K. M. Olvey, and J. J. Bartko, "Nerve and tendon gliding exercises and the conservative management of carpal tunnel syndrome," J. Hand Ther., vol. 11, no. 3, pp. 171-179, Jul. 1998.

[15] K. Lohse, N. Shirzad, A. Verster, N. Hodges, and H. F. M. Van der Loos, "Video Games and Rehabilitation," J. Neurol. Phys. Ther., vol. 37, no. 4, pp. 166-175, Dec. 2013.

[16] F. Anderson, M. Annett, and W. F. Bischof, "Lean on Wii: Physical rehabilitation with virtual reality Wii peripherals," Stud. Health Technol. Inform., vol. 154, pp. 229-234, 2010.

[17] A. Y. Wang, "Games for Physical Therapy," SIGCHI Conf., pp. 4-8, 2012.

[18] M. E. Kho, A. Damluji, J. M. Zanni, and D. M. Needham, "Feasibility and observed safety of interactive video games for physical rehabilitation in the intensive care unit: a case series," J. Crit. Care, vol. 27 , no. 2, p. 219.e1-219.e6, Apr. 2012.

[19] A. de Mauro, "Virtual Reality Based Rehabilitation and Game Technology," in 1st International Workshop 
on Engineering Interactive Computing Systems for Medicine and Health Care (EICSMed 2011), co-located with the ACM SIGCHI Symposium on Engineering Interactive Computing Systems (EICS 2011), 2011, pp. 48-52.

[20] M. Khademi, H. Mousavi Hondori, A. McKenzie, L. Dodakian, C. V. Lopes, and S. C. Cramer, "Free-hand interaction with leap motion controller for stroke rehabilitation," in Proceedings of the extended abstracts of the 32nd annual ACM conference on Human factors in computing systems - CHI EA '14, 2014, pp. 16631668.

[21] L. Geurts, V. Vanden Abeele, J. Husson, F. Windey, M. Van Overveldt, J.-H. Annema, and S. Desmet, "Digital games for physical therapy: fulfilling the need for calibration and adaptation," in Proceedings of the fifth international conference on Tangible, embedded, and embodied interaction - TEl '11, 2011, vol. 53, p. 117.

[22] "Virtualrehab." [Online]. Available: http://www.virtualrehab.info/.

[23] F. Weichert, D. Bachmann, B. Rudak, and D. Fisseler, "Analysis of the Accuracy and Robustness of the Leap Motion Controller," Sensors, vol. 13, no. 5, pp. 6380-6393, May 2013.

[24] "Leap Motion API Reference." [Online]. Available: https://developer.leapmotion.com/documentation/csharp/api/Leap_Classes.html?proglang=csharp. [Accessed: 05-Mar-2016].

[25] J. Bae and A. Kim, "Design and Development of Unity3D Game Engine-Based Smart SNG (Social Network Game)," Int. J. Multimed. Ubiquitous Eng., vol. 9, no. 8, pp. 261-266, Aug. 2014.

[26] S. Wang, Z. Mao, C. Zeng, H. Gong, S. Li, and B. Chen, "A new method of virtual reality based on Unity3D," 2010 18th Int. Conf. Geoinformatics, pp. 1-5, 2010.

[27] I. Pachoulakis and D. Tsilidi, "Technology-assisted Carpal Tunnel Syndrome Rehabilitation using serious games: the Roller Ball example," Adv. Image Video Process., vol. 4, no. 4, Aug. 2016. 